\title{
Magnetic Properties and Flux Distribution in the LaFeCoSi/FeCoV Hybrid Structure
}

\author{
R. Gozdur ${ }^{a, *}$, M. LeBIOdA $^{b}$ AND M. NAJGeBAuer ${ }^{c}$ \\ ${ }^{a}$ Lodz University of Technology, Department of Semiconductors and Optoelectronic Devices, \\ Wolczanska 211/215, 90-924 Lodz, Poland \\ ${ }^{b}$ Lodz University of Technology, Institute of Electrical Engineering Systems, \\ St. Stefanowskiego 18/22, 90-924 Lodz, Poland \\ ${ }^{c}$ Czestochowa University of Technology, Faculty of Electrical Engineering, \\ Armii Krajowej 17, 42-200 Czestochowa, Poland
}

\begin{abstract}
The study contains a description of magnetic properties of LaFeCoSi/FeCoV structure. The hybrid structure contains magnetocaloric LaFeCoSi core with high-induction ferromagnetic laminations. The magnetic field inside the magnetic structure is gained by the fringe field from FeCoV laminations. Results of modelling and experimental investigations of modified magnetic properties of $\mathrm{LaFeCoSi} / \mathrm{FeCoV}$ structure were presented and discussed in the paper.
\end{abstract}

DOI: 10.12693/APhysPolA.131.1294

PACS/topics: $75.30 . \mathrm{Sg}, 75.30 . \mathrm{Kz}, 75.50 . \mathrm{Bb}$

\section{Introduction}

The discovery of the giant magnetocaloric effect in 1995 resulted in the growth of interest in magnetocaloric materials. It stems from the fact that the magnetocaloric effect (MCE) can be used for magnetic refrigeration in the room temperature. Currently, several types of magnetocaloric materials (MCM) are intensively developed and frequently used in prototype magnetic refrigerators. Among these materials are compounds of $\mathrm{Gd}-$ Ge-Si [1], $\mathrm{MnFe}(\mathrm{P}, \mathrm{Si})$ [2], $\mathrm{MnFe}(\mathrm{P}, \mathrm{As})$ [3], La-Fe-Si [4], $\mathrm{La}(\mathrm{Fe}, \mathrm{Si}) \mathrm{H}[5,6]$, $\mathrm{La}(\mathrm{Fe}, \mathrm{Co}) \mathrm{Si}[7-9]$ and $\mathrm{La}(\mathrm{Fe}, \mathrm{Mn}) \mathrm{Si}-$ $\mathrm{H}$ [10]. The newly emerging solutions use La-Fe-Sior $\mathrm{Mn}-\mathrm{Fe}-\mathrm{P}$ compounds due to their entropy changes $\left|\Delta S_{M A X}\right|=(10 \div 25) \mathrm{J} \mathrm{kg}^{-1} \mathrm{~K}^{-1}$ and the adiabatic temperature changes $\Delta T_{A D}=(5 \div 7) \mathrm{K}$ in a magnetic field $\mu_{0} H$ less than $2 \mathrm{~T}$ [11]. The above-mentioned peak values of the parameters $\left|\Delta S_{M A X}\right|$ and $\Delta T_{A D}$ are reached only in a small operating temperature range in immediate vicinity of the Curie temperature of magnetocaloric material. Another limitation is frequently referred to the actual value of the magnetic field when permanent magnet systems are used as a source of magnetic field. Typically, an achievable magnetic field below $1 \mathrm{~T}$ is still sufficient to obtain heat transport with the adiabatic temperature change $\Delta T_{A D}$ more than $1 \mathrm{~K}$ [11]. In that case, MCE is much sensitive to operating temperature of MCM, spatial distribution of magnetic field, frequency of refrigeration cycle and other factors [12]. Temperature and frequency are strictly operating parameters while the effective value and spatial distribution of the magnetic field are design

*corresponding author; e-mail: gozdur@p.lodz.pl parameters $[13,14]$. Improved spatial uniformity of the magnetic field in the regenerator's bed may indirectly improve the relative cooling power (RCP) of the magnetocaloric refrigeration cycle $[11,12]$. Considered changes can be introduced by the use of additional ferromagnetic layers (FM) having a substantially higher Curie temperature $T_{\mathrm{C}}$. This paper presents the study on magnetic properties of the hybrid structure in the FM/MCM/FM configuration. Ferromagnetic laminates FeCoV (Vacuumschmelze commercial grade: Vacoflux 50) and a magnetocaloric sintered powder LaFeCoSi (Vacuumschmelze commercial grade: Calorivac C) were used for testing. The evaluation of magnetic properties of the structure was carried out on the basis of measurements and the magnetic field distribution has been estimated from FEM analysis.

\section{Hybrid structure and instrumentation}

The test structure was made as a laminated closed magnetic circuit in the shape of a square (Fig. 1). The magnetocaloric core was assembled of four uniform plates which were made of the $\mathrm{LaFe}_{10.8} \mathrm{Co}_{1.1} \mathrm{Si}_{1.1}$ (Calorivac C) sintered powder with a defined Curie temperature of $300 \mathrm{~K}$. The overall dimensions of the applied plates were $40 \times 17 \times 5 \mathrm{~mm}^{3}$ respectively, and the total mass of the magnetocaloric core equals $m_{M C M}=94.10 \mathrm{~g}$. The outer surfaces of the test core were covered with ferromagnetic (FM) laminations of the $\mathrm{Fe}_{49.1} \mathrm{Co}_{49.0} \mathrm{~V}_{1.9}$ (Vacoflux 50). The total thickness of a single FM lamination was $400 \mu \mathrm{m}$ and the total weight of all laminations in the structure was $15.98 \mathrm{~g}$. The cross-sectional ratio of FM/MCM surfaces defined as $\gamma_{s}=s_{F M} / s_{M C M}$ was on the level of 0.16 .

Schematic diagram (Fig. 1) shows the assembling method of the structure in the sample holder, windings 


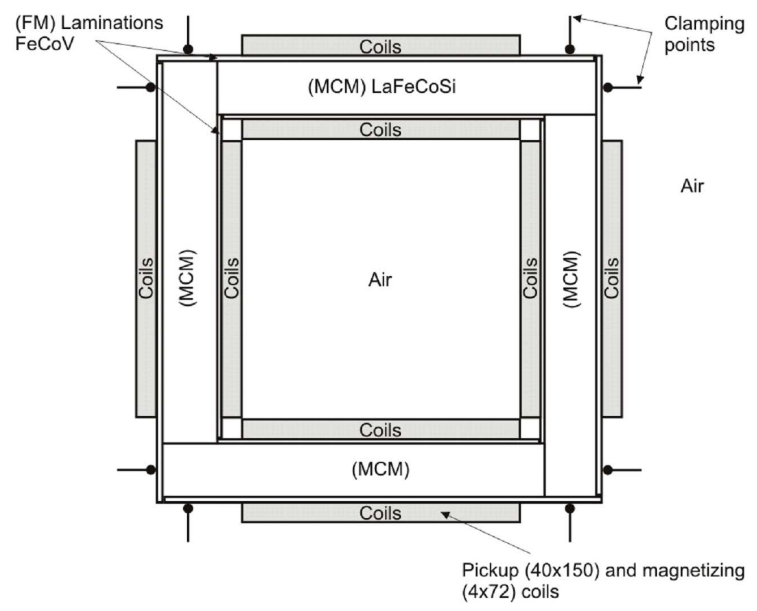

Fig. 1. Diagram of the tested structure with FM laminations and MCM core.

and clamping system to reduce gaps and magnetic reluctance between FM and MCM components.

Measurements of magnetic parameters were performed under adiabatic conditions. A compact thermostatic chamber equipped with a thermoelectric heat pump (nominal power of $98 \mathrm{~W}$ ) and LTC1923 controller was used for the thermal control of the tested structure. Long-term stability and accuracy of the setpoints was less than $0.2 \mathrm{~K}$ in the temperature range from $280 \mathrm{~K}$ to $350 \mathrm{~K}$ for the settling time of $3600 \mathrm{~s}$.

Hysteresis loops, magnetic parameters $J_{\max }, H_{\max }, B_{r}$, $H_{c}$ and their temperature dependences were measured in accordance to IEC 60404-06: 2004. Applied instrumentation and the measurement system were elaborated on another paper [15]. Power losses $P_{s}$ were measured with the usage of the unbalanced bridge method (UBM) [16]. The UBM is a more accurate method in the case of measurements of ultra-low power losses resulting from the quasi-static magnetization $f_{\text {mag }}=1 \mathrm{~Hz}$ and magnetic properties of the structure in the magnetic phase transition.

\section{Magnetic properties of the FM/MCM/FM structure}

The modified magnetic properties of the hybrid structure consisting of two materials with different Curie temperatures were tested in the temperature range including the Curie temperature $T_{\mathrm{C}}=300 \mathrm{~K}$ of the magnetocaloric material. In the range from $292 \mathrm{~K}$ to $350 \mathrm{~K}$ measured magnetic polarization $J_{\max }$ in the hybrid structure drops to the value of $0.43 \mathrm{~T}$ while reduced polarization $J_{\max }$ in MCM core without FM lamination does not exceed $0.03 \mathrm{~T}$. The influence of the temperature on magnetic properties of the magnetocaloric compound $\mathrm{LaFe}_{10.8} \mathrm{Co}_{1.1} \mathrm{Si}_{1.1}$ was discussed in paper [17]. The same dependences of the FM laminations in the considered temperature range can be neglected because the Curie temperature equals $1223 \mathrm{~K}$, respectively. Table I shows key physical parameters of used materials.

TABLE I

Parameters of magnetic components in the hybrid structure.

\begin{tabular}{c|c|c}
\hline \hline Parameter & Vacoflux 50 & Calorivac C \\
\hline$J_{S A T}[\mathrm{~T}]$ & 2.25 & 0.75 \\
$\mu_{r} \max$ & 6200 & 1000 \\
$P_{s}[\mathrm{~mW} / \mathrm{kg}]$ & 104 & 8 \\
$(T=300 \mathrm{~K}$, & & \\
$\left.f_{\text {mag }}=1 \mathrm{~Hz}\right)$ & & \\
$\rho\left[\mathrm{kg} / \mathrm{m}^{3}\right]$ & 8120 & 7288 \\
$T_{\mathrm{C}}[\mathrm{K}]$ & 1233 & 300
\end{tabular}

The test hybrid structure remains in the ferromagnetic state above the temperature of $300 \mathrm{~K}$, while MCM core without FM laminations exhibits paramagnetic properties (Fig. 2a,b).
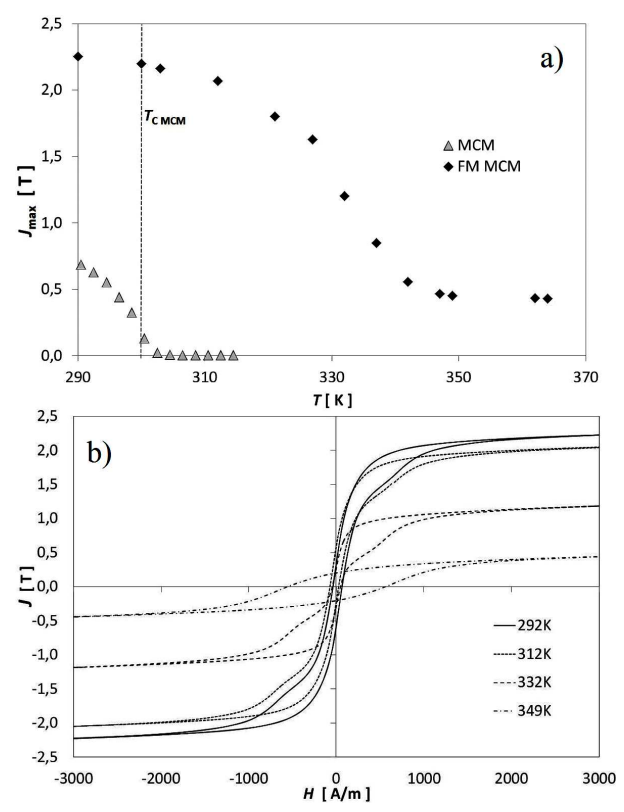

Fig. 2. Influence of temperature on peak magnetic polarization $J_{\max }($ a) and the shape of the quasistatic hysteresis loops at given frequency $f_{m a g}=1 \mathrm{~Hz}(\mathrm{~b})$.

Significant decrease of the saturation $J_{\max }$ within the hybrid structure (Fig. 2a,b) is shifted towards the temperature of $340 \mathrm{~K}$ and remains relatively high. Collapse of the saturation is strictly connected with the magnetic phase transition in the MCM core. Descending permeability in the MCM components causes gaps between the components of the structure and reduces average polarization. The decrease in the spatial distribution of the polarization in the structure is twice smaller than it is in the MCM without FM laminations. The measurements indicate that the field distribution and the resultant magnetic parameters of the structure at a temperature above $T_{\mathrm{C}}$ depends considerably on the parameters of FM. In 
the immediate vicinity of the magnetic phase transition, the influence of the FM on the magnetic properties is dominant (Fig. 3a,b).

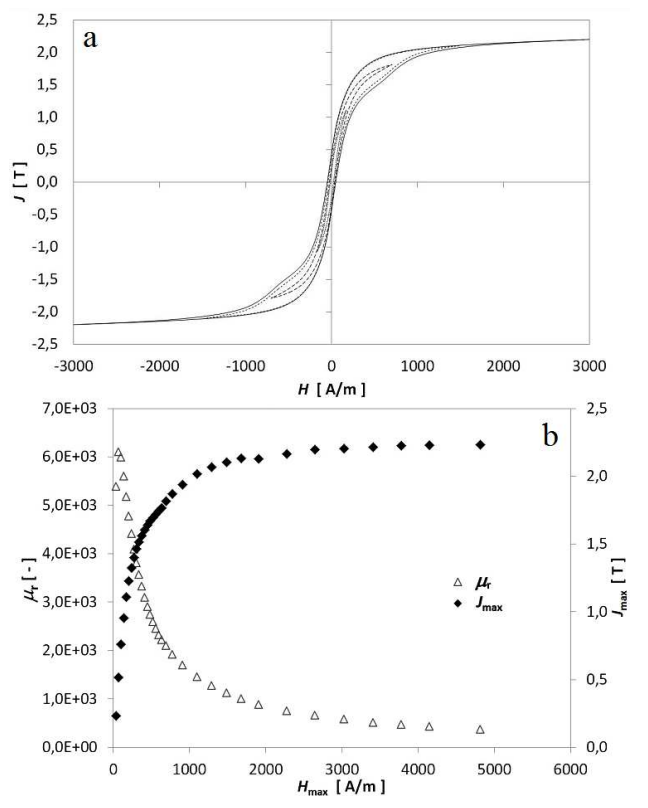

Fig. 3. Set of minor hysteresis loops (a), permeability and initial magnetization curves (b) of the hybrid structure at given temperature $T=300 \mathrm{~K}$ and $f_{m a g}=1 \mathrm{~Hz}$.
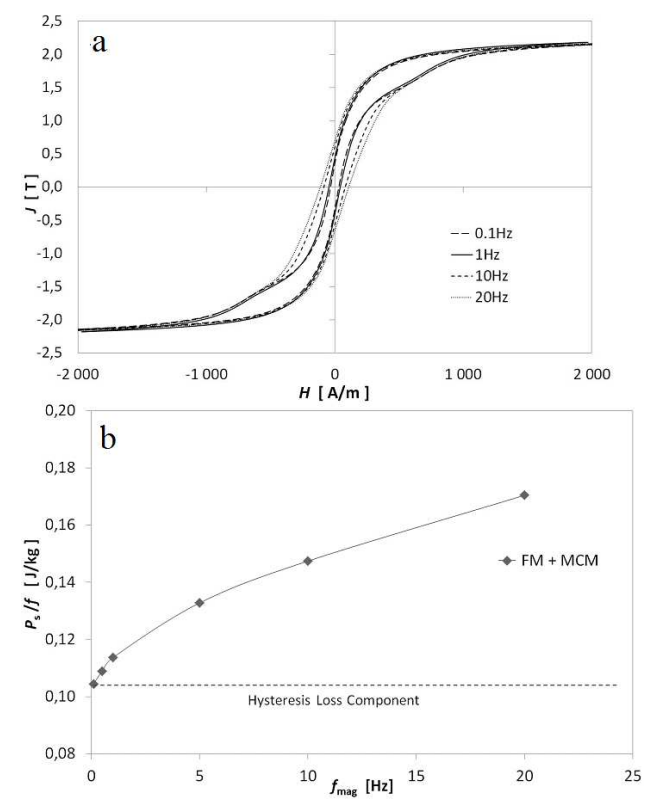

Fig. 4. Influence of magnetizing frequency $f_{\text {mag }}$ on hysteresis loops (a) and total energy loss per cycle (b) at given temperature $T=300 \mathrm{~K}$.

Ranging from $1.5 \mathrm{~T}$ till $2.0 \mathrm{~T}$, the shape of the hysteresis loop $J(H)$ is significantly changed (Figs. 2b, 3a). This is the result of saturation in the MCM components and causes a significant increase in energy loss per cycle (Fig. 4b) compared to the loss in the MCM core without FM laminations [17]. Coercive field and magnetic remanence of the hybrid structure (Fig. 3a) do not have an impact on the hysteresis loss component. Nevertheless, the local distortion in the shape of hysteresis loops observed in the considered range 1.5-2.0 T (Fig. 4a) does not depend on the frequency $f_{m a g}$. It also results in growth of the hysteresis loss component [17].

\section{Magnetic flux distribution inside the structure}

The experimental results show that the process of magnetization of the structure undergoes a significant change upon addition of FM laminations. Differences in magnetic parameters of applied materials translate directly into changes in distribution of magnetic polarization in the structure, even at low ratio $\gamma_{s}$. In order to analyze the local distribution of the magnetic polarization, a twodimensional FEM model with a geometry corresponding to the configuration of the test structure was developed. A numerical analysis was conducted in the Comsol software which provides tools for modeling of multiphysics nonlinear problems [18-20].

The model was submitted for steady state analysis with preservation of the dimensions of the tested structure (Fig. 1) and experimental quasistatic studies at a $f_{\text {mag }}$ frequency of $1 \mathrm{~Hz}$. Non-magnetic components of the sample holder and windings were predefined in software accordingly to common relations $J=f(H)$. Magnetic components of the hybrid structure were defined by the data from measurements (Fig. 5) and Vacuumschmelze technical notes.

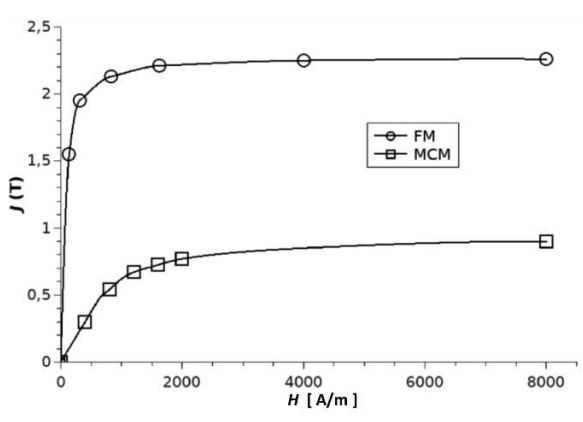

Fig. 5. Initial magnetization curves of the magnetocaloric (MCM) components and ferromagnetic laminations (FM) applied in the FEM model.

Significant differences in the parameters of non-linear magnetic materials determine the distribution of the magnetic field in the structure and translate into a change in the average polarization. Experimental tests do not give the possibility of carrying out the analysis of the local magnetic field distribution in the structure. In order to observe the presence of FM in the structure, a separate numerical analysis of the homogeneous MCM structure and the hybrid one was carried out. 


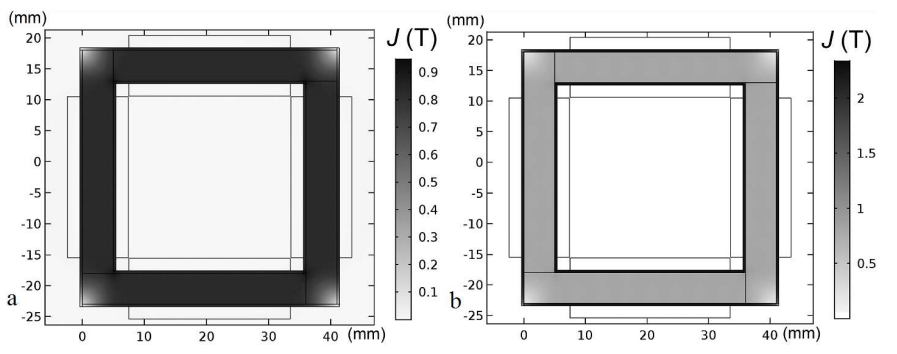

Fig. 6. FEM simulations of the magnetic field distribution in the homogeneous MCM structure (a) and the investigated FM/MCM structure (b).

Thin FM laminations disposed on the inner and outer wall of MCM plates lead to the unification of magnetic field distribution in the structure, especially at its corners (Fig. 6a,b). Changes in the polarization density at regular parts of the structure are small (Fig. 7). This is due to the disparities in the volume and permeability. At a low magnetic field strength $H$ of several hundred $\mathrm{A} / \mathrm{m}$, magnetic polarization approaches $2 \mathrm{~T}$ in the $\mathrm{FM}$, which is the point of inflection in the initial magnetization curve $J=f(H)$. It is very important that the FM laminations achieve pre-saturation thus a further increase in $H$ leads to the uniformity of a magnetic field distribution in other parts of the structure. This effect is noticeable as the distortion in the shape of hysteresis loops (Figs. 2a, 3a, 4a).

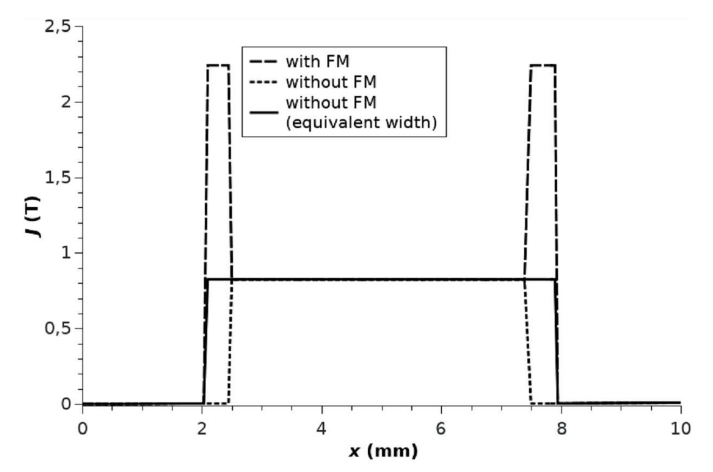

Fig. 7. Magnetic polarization distribution in the crosssection of the hybrid structure (FEM analysis).

However, simplifying the shape of the structure does not illustrate the full significance of the FM laminations in the real magnetic refrigerators because of more complex shapes of magnetocaloric beds. Heightened importance of the FM layers on the surfaces of the magnetocaloric beds should be visible for irregular comb shapes of beds with a lot of corners.

\section{Summary}

The study of LaFeCoSi/FeCoV structure confirms strong influence of $\mathrm{FeCoV}$ ferromagnetic laminations on resultant magnetic properties and magnetic field distribution inside magnetocaloric components. The hybrid structure exhibits ferromagnetic properties at temperatures above Curie temperatures of the magnetocaloric core. Furthermore, the presence of FM laminations adjusts the spatial distribution of the magnetic flux. FEM analysis indicates that the most important changes are in the corners and near the FM-MCM contacts.

\section{References}

[1] V.K. Pecharsky, K.A. Gschneidner Jr., Phys. Rev. Lett. 78, 4494 (1997).

[2] N.H. Dung, L. Zhang, Z.Q. Ou, E. Brück, Scr. Mater. 67, 975 (2012).

[3] E. Brück, M. Ilyn, M. Tishin, O. Tegus, J. Magn. Magn. Mater. 290-291, 8 (2005).

[4] S. Fujieda, A. Fujita, K. Fukamichi, Appl. Phys. Lett. 81, 1276 (2002).

[5] A. Fujita, S. Fujieda, Y. Hasegawa, K. Fukamichi, Phys. Rev. B 67, 104416 (2003).

[6] A. Barcza, V. Zellmann, M. Zapf, T. Elwert, P. Sommer, M. Katter, IEEE Trans. Magn. 48, 4066 (2012).

[7] M. Katter, V. Zellmann, G.W. Reppel, K. Uestuener, IEEE Trans. Magn. 44, 3044 (2008).

[8] R. Bjørk, C.R.H. Bahl, M. Katter, J. Magn. Magn. Mater. 322, 3882 (2010).

[9] P. Gębara, P. Pawlik, B. Michalski, J.J. Wysłocki, Acta Phys. Pol. A 127, 576 (2015).

[10] A. Barcza, M. Katter, V. Zellmann, S. Russek, S. Jacobs, C. Zimm, IEEE Trans. Magn. 47, 3391 (2011).

[11] K.G. Sandeman, Scr. Mater. 67, 566 (2012).

[12] A.M. Tishin, Y.I. Spichkin, The Magnetocaloric Effect and Its Applications, Institute of Physics Publ. Bristol 2003.

[13] Y. You, Y. Guo, S. Xiao, S. Yu, H. Ji, X. Luo, J. Magn. Magn. Mater. 405, 231 (2016).

[14] R. Bjørk, C.R.H. Bahl, A. Smith, D.V. Christensen, N. Pryds, J. Magn. Magn. Mater. 322, 3324 (2010).

[15] R. Gozdur, M. Najgebauer, J. Electr. Eng. 66, 37 (2015).

[16] R. Gozdur, A. Majocha, Prz. Elektrotechn. 86, 79 (2010) (in Polish).

[17] R. Gozdur, M. Lebioda, Ł. Bernacki, Acta Phys. Pol. A 128, 98 (2015).

[18] J. Rymaszewski, M. Lebioda, E. Korzeniewska, Prz. Elektrotechn. 88, 183 (2012) (in Polish).

[19] R. Pawlak, M. Tomczyk, M. Walczak, J. Mizeraczyk, M. Tański, K. Garasz, Microelectron. Eng. 151, 47 (2016).

[20] G. Paoli, O. Biro, G. Buchgraber, IEEE Trans. Magn. 34, 2625 (1998). 